\title{
Stability of quadratic functional equations in tempered distributions
}

\section{Young-Su Lee*}

\section{"Correspondence:} masuri@sogang.ac.kr Department of Mathematics, Sogang University, Seoul, 121-741, Republic of Korea

\begin{abstract}
We reformulate the following quadratic functional equation:

$$
f(k x+y)+f(k x-y)=2 k^{2} f(x)+2 f(y)
$$

as the equation for generalized functions. Using the fundamental solution of the heat equation, we solve the general solution of this equation and prove the Hyers-Ulam stability in the spaces of tempered distributions and Fourier hyperfunctions.
\end{abstract}

Keywords: quadratic functional equation; stability; tempered distribution; heat kernel; Gauss transform

\section{Introduction}

In 1940, Ulam [31] raised a question concerning the stability of group homomorphisms as follows:

Let $G_{1}$ be a group and let $G_{2}$ be a metric group with the metric $d(\cdot, \cdot)$. Given $\epsilon>0$, does there exist a $\delta>0$ such that if a function $h: G_{1} \rightarrow G_{2}$ satisfies the inequality $d(h(x y), h(x) h(y))<\delta$ for all $x, y \in G_{1}$, then there exists a homomorphism $H: G_{1} \rightarrow G_{2}$ with $d(h(x), H(x))<\epsilon$ for all $x \in G_{1}$ ?

The case of approximately additive mappings was solved by Hyers [16] under the assumption that $G_{2}$ is a Banach space. In 1978, Rassias [25] generalized Hyers' result to the unbounded Cauchy difference.

During the last decades, stability problems of various functional equations have been extensively studied and generalized by a number of authors (see $[13,14,17,19,24,27$, 30]). In particular, the stability problem of the following quadratic functional equation

$$
f(x+y)+f(x-y)=2 f(x)+2 f(y)
$$

was proved by Skof [29]. Thereafter, many authors studied the stability problems of (1.1) in various settings (see $[3,4,12,18]$ ). Usually, quadratic functional equations are used to characterize the inner product spaces. Note that a square norm on an inner product space satisfies the parallelogram equality

$$
\|x+y\|^{2}+\|x-y\|^{2}=2\|x\|^{2}+2\|y\|^{2}
$$

(0) 2012 Lee; licensee Springer. This is an Open Access article distributed under the terms of the Creative Commons Attribution License (http://creativecommons.org/licenses/by/2.0), which permits unrestricted use, distribution, and reproduction in any medium, provided the original work is properly cited. 
for all vectors $x, y$. By virtue of this equality, the quadratic functional equation (1.1) is induced. It is well known that a function $f$ between real vector spaces satisfies (1.1) if and only if there exists a unique symmetric biadditive function $B$ such that $f(x)=B(x, x)$ (see $[1,13,17,19,27])$. The biadditive function $B$ is given by

$$
B(x, y)=\frac{1}{4}(f(x+y)-f(x-y)) .
$$

Recently, Lee et al. [21] introduced the following quadratic functional equation which is equivalent to (1.1):

$$
f(k x+y)+f(k x-y)=2 k^{2} f(x)+2 f(y),
$$

where $k$ is a fixed positive integer. They proved the Hyers-Ulam-Rassias stability of this equation in Banach spaces. Wang [32] considered the intuitionistic fuzzy stability of (1.2) by using the fixed-point alternative. Saadati and Park [26] proved the Hyers-Ulam-Rassias stability of (1.2) in non-Archimedean $\mathcal{L}$-fuzzy normed spaces.

In this paper, we solve the general solution and the stability problem of (1.2) in the spaces of generalized functions such as $\mathcal{S}^{\prime}$ of tempered distributions and $\mathcal{F}^{\prime}$ of Fourier hyperfunctions. Using pullbacks, Chung and Lee [8] reformulated (1.1) as the equation for generalized functions and proved that every solution of (1.1) in $\mathcal{S}^{\prime}$ (or $\mathcal{F}^{\prime}$, resp.) is a quadratic form. Also, Chung $[7,11]$ proved the stability problem of (1.1) in the spaces $\mathcal{S}^{\prime}$ and $\mathcal{F}^{\prime}$. Making use of the similar methods as in [7-11,22], we reformulate (1.2) and the related inequality in the spaces of generalized functions as follows:

$$
\begin{aligned}
& u \circ A+u \circ B=2 k^{2} u \circ P+2 u \circ Q, \\
& \left\|u \circ A+u \circ B-2 k^{2} u \circ P-2 u \circ Q\right\| \leq \epsilon,
\end{aligned}
$$

where $A, B, P$, and $Q$ are the functions defined by

$$
A(x, y)=k x+y, \quad B(x, y)=k x-y, \quad P(x, y)=x, \quad Q(x, y)=y .
$$

Here, $\circ$ denotes the pullback of generalized functions and the inequality $\|v\| \leq \epsilon$ in (1.4) means that $|\langle v, \varphi\rangle| \leq \epsilon\|\varphi\|_{L^{1}}$ for all test functions $\varphi$. We refer to [15] for pullbacks and to $[2,7-11]$ for more details of the spaces of generalized functions.

As results, we shall prove that every solution $u$ in $\mathcal{S}^{\prime}$ (or $\mathcal{F}^{\prime}$, resp.) of Eq. (1.3) is a quadratic form

$$
u=\sum_{1 \leq i \leq j \leq n} a_{i j} x_{i} x_{j},
$$

where $a_{i j} \in \mathbb{C}$. Also, we shall prove that every solution $u$ in $\mathcal{S}^{\prime}$ (or $\mathcal{F}^{\prime}$, resp.) of the inequality (1.4) can be written uniquely in the form

$$
u=\sum_{1 \leq i \leq j \leq n} a_{i j} x_{i} x_{j}+\mu(x),
$$


where $\mu$ is a bounded measurable function such that

$$
\|\mu\|_{L^{\infty}} \leq \begin{cases}\frac{\epsilon}{2}, & k=1, \\ \frac{\left(k^{2}+1\right) \epsilon}{2 k^{2}\left(k^{2}-1\right)}, & k \geq 2 .\end{cases}
$$

\section{Preliminaries}

In this section, we introduce the spaces of tempered distributions and Fourier hyperfunctions. Here, we use the $n$-dimensional notations. If $\alpha=\left(\alpha_{1}, \ldots, \alpha_{n}\right) \in \mathbb{N}_{0}^{n}$, where $\mathbb{N}_{0}^{n}$ is the set of nonnegative integers, then $|\alpha|=\alpha_{1}+\cdots+\alpha_{n}, \alpha !=\alpha_{1} ! \cdots \alpha_{n}$ !. For $x=\left(x_{1}, \ldots, x_{n}\right) \in \mathbb{R}^{n}$, we denote $x^{\alpha}=x_{1}^{\alpha_{1}} \cdots x_{n}^{\alpha_{n}}$ and $\partial^{\alpha}=\left(\partial / \partial x_{1}\right)^{\alpha_{1}} \cdots\left(\partial / \partial x_{n}\right)^{\alpha_{n}}$.

\subsection{Tempered distributions}

We present a very useful space of test functions for the tempered distributions as follows.

Definition $2.1([15,28])$ An infinitely differentiable function $\varphi$ in $\mathbb{R}^{n}$ is called rapidly decreasing if

$$
\|\varphi\|_{\alpha, \beta}=\sup _{x \in \mathbb{R}^{n}}\left|x^{\alpha} \partial^{\beta} \varphi(x)\right|<\infty
$$

for all $\alpha, \beta \in \mathbb{N}_{0}^{n}$. The vector space of such functions is denoted by $\mathcal{S}\left(\mathbb{R}^{n}\right)$. A linear functional $u$ on $\mathcal{S}\left(\mathbb{R}^{n}\right)$ is said to be a tempered distribution if there exists the constant $C \geq 0$ and the nonnegative integer $N$ such that

$$
|\langle u, \varphi\rangle| \leq C \sum_{|\alpha|,|\beta| \leq N} \sup _{x \in \mathbb{R}^{n}}\left|x^{\alpha} \partial^{\beta} \varphi\right|
$$

for all $\varphi \in \mathcal{S}\left(\mathbb{R}^{n}\right)$. The set of all tempered distributions is denoted by $\mathcal{S}^{\prime}\left(\mathbb{R}^{n}\right)$.

We note that, if $\varphi \in \mathcal{S}\left(\mathbb{R}^{n}\right)$, then each derivative of $\varphi$ decreases faster than $|x|^{-N}$ for all $N>0$ as $|x| \rightarrow \infty$. It is easy to see that the function $\varphi(x)=\exp \left(-a|x|^{2}\right)$, where $a>0$ belongs to $\mathcal{S}\left(\mathbb{R}^{n}\right)$, but $\psi(x)=\left(1+|x|^{2}\right)^{-1}$ is not a member of $\mathcal{S}\left(\mathbb{R}^{n}\right)$. It is known from [5] that (2.1) is equivalent to

$$
\sup _{x \in \mathbb{R}^{n}}\left|x^{\alpha} \varphi(x)\right|<\infty, \quad \sup _{\xi \in \mathbb{R}^{n}}\left|\xi^{\beta} \hat{\varphi}(\xi)\right|<\infty
$$

for all $\alpha, \beta \in \mathbb{N}_{0}^{n}$, where $\hat{\varphi}$ is the Fourier transform of $\varphi$.

For example, every polynomial $p(x)=\sum_{|\alpha| \leq m} a_{\alpha} x^{\alpha}$, where $a_{\alpha} \in \mathbb{C}$, defines a tempered distribution by

$$
\langle p(x), \varphi\rangle=\int_{\mathbb{R}^{n}} p(x) \varphi(x) d x, \quad \varphi \in \mathcal{S}\left(\mathbb{R}^{n}\right) .
$$

Note that tempered distributions are generalizations of $L^{p}$-functions. These are very useful for the study of Fourier transforms in generality, since all tempered distributions have a Fourier transform. 


\subsection{Fourier hyperfunctions}

Imposing the growth condition on $\|\cdot\|_{\alpha, \beta}$ in (2.1) Sato and Kawai introduced the new space of test functions for the Fourier hyperfunctions as follows.

Definition $2.2([6])$ We denote by $\mathcal{F}\left(\mathbb{R}^{n}\right)$ the set of all infinitely differentiable functions $\varphi$ in $\mathbb{R}^{n}$ such that

$$
\|\varphi\|_{A, B}=\sup _{x, \alpha, \beta} \frac{\left|x^{\alpha} \partial^{\beta} \varphi(x)\right|}{A^{|\alpha|} B^{|\beta|} \alpha ! \beta !}<\infty
$$

for some positive constants $A, B$ depending only on $\varphi$. The strong dual of $\mathcal{F}\left(\mathbb{R}^{n}\right)$, denoted by $\mathcal{F}^{\prime}\left(\mathbb{R}^{n}\right)$, is called the Fourier hyperfunction.

It can be verified that the seminorm (2.2) is equivalent to

$$
\|\varphi\|_{h, k}=\sup _{x, \alpha} \frac{\left|\partial^{\alpha} \varphi(x)\right| \exp k|x|}{h^{|\alpha|} \alpha !}<\infty
$$

for some constants $h, k>0$. Furthermore, it is shown in [6] that (2.2) is equivalent to

$$
\sup _{x \in \mathbb{R}^{n}}|\varphi(x)| \exp k|x|<\infty, \quad \sup _{\xi \in \mathbb{R}^{n}}|\hat{\varphi}(\xi)| \exp h|\xi|<\infty
$$

for some $h, k>0$.

Fourier hyperfunctions were introduced by Sato in 1958. The space $\mathcal{F}^{\prime}\left(\mathbb{R}^{n}\right)$ is a natural generalization of the space $\mathcal{S}^{\prime}\left(\mathbb{R}^{n}\right)$ and can be thought informally as distributions of a infinite order. Observing the above growth conditions, we can easily see the following topological inclusions:

$$
\mathcal{F}\left(\mathbb{R}^{n}\right) \hookrightarrow \mathcal{S}\left(\mathbb{R}^{n}\right), \quad \mathcal{S}^{\prime}\left(\mathbb{R}^{n}\right) \hookrightarrow \mathcal{F}^{\prime}\left(\mathbb{R}^{n}\right) .
$$

\section{General solution in generalized functions}

In order to solve the general solution of (1.3), we employ the $n$-dimensional heat kernel, fundamental solution of the heat equation,

$$
E_{t}(x)= \begin{cases}(4 \pi t)^{-n / 2} \exp \left(-|x|^{2} / 4 t\right), & x \in \mathbb{R}^{n}, t>0, \\ 0, & x \in \mathbb{R}^{n}, t \leq 0 .\end{cases}
$$

Since for each $t>0, E_{t}(\cdot)$ belongs to the space $\mathcal{F}\left(\mathbb{R}^{n}\right)$, the convolution

$$
\tilde{u}(x, t)=\left(u * E_{t}\right)(x)=\left\langle u_{y}, E_{t}(x-y)\right\rangle
$$

is well defined for all $u$ in $\mathcal{F}^{\prime}\left(\mathbb{R}^{n}\right)$, which is called the Gauss transform of $u$. Subsequently, the semigroup property

$$
\left(E_{t} * E_{s}\right)(x)=E_{t+s}(x)
$$

of the heat kernel is very useful to convert Eq. (1.3) into the classical functional equation defined on upper-half plane. We also use the following famous result, the so-called heat kernel method, which is stated as follows. 
Theorem 3.1 ([23]) Let $u \in \mathcal{S}^{\prime}\left(\mathbb{R}^{n}\right)$. Then its Gauss transform $\tilde{u}$ is a $C^{\infty}$-solution of the heat equation

$$
(\partial / \partial t-\Delta) \tilde{u}(x, t)=0
$$

satisfying

(i) There exist positive constants $C, M$, and $N$ such that

$$
|\tilde{u}(x, t)| \leq C t^{-M}(1+|x|)^{N} \quad \text { in } \mathbb{R}^{n} \times(0, \delta) .
$$

(ii) $\tilde{u}(x, t) \rightarrow$ uas $t \rightarrow 0^{+}$in the sense that for every $\varphi \in \mathcal{S}\left(\mathbb{R}^{n}\right)$,

$$
\langle u, \varphi\rangle=\lim _{t \rightarrow 0^{+}} \int \tilde{u}(x, t) \varphi(x) d x .
$$

Conversely, every $C^{\infty}$-solution $U(x, t)$ of the heat equation satisfying the growth condition (3.1) can be uniquely expressed as $U(x, t)=\tilde{u}(x, t)$ for some $u \in \mathcal{S}^{\prime}\left(\mathbb{R}^{n}\right)$.

Similarly, we can represent Fourier hyperfunctions as a special case of the results as in [20]. In this case, the estimate (3.1) is replaced by the following:

For every $\epsilon>0$, there exists a positive constant $C_{\epsilon}$ such that

$$
|\tilde{u}(x, t)| \leq C_{\epsilon} \exp (\epsilon(|x|+1 / t)) \quad \text { in } \mathbb{R}^{n} \times(0, \delta) .
$$

Here, we need the following lemma to solve the general solution of (1.3).

Lemma 3.2 Suppose that $f: \mathbb{R}^{n} \times(0, \infty) \rightarrow \mathbb{C}$ is a continuous function satisfying the equation

$$
f\left(k x+y, k^{2} t+s\right)+f\left(k x-y, k^{2} t+s\right)=2 k^{2} f(x, t)+2 f(y, s)
$$

for all $x, y \in \mathbb{R}^{n}, t, s>0$. Then the solution $f$ is the quadratic-additive function

$$
f(x, t)=\sum_{1 \leq i \leq j \leq n} a_{i j} x_{i} x_{j}+b t
$$

for some $a_{i j}, b \in \mathbb{C}$.

Proof Define a function $h: \mathbb{R}^{n} \times(0, \infty) \rightarrow \mathbb{C}$ as $h(x, t):=f(x, t)-f(0, t)$. We immediately have $h(0, t)=0$ and

$$
h\left(k x+y, k^{2} t+s\right)+h\left(k x-y, k^{2} t+s\right)=2 k^{2} h(x, t)+2 h(y, s)
$$

for all $x, y \in \mathbb{R}^{n}, t, s>0$. Putting $y=0$ in (3.3) yields

$$
h\left(k x, k^{2} t+s\right)=k^{2} h(x, t)
$$


for all $x \in \mathbb{R}^{n}, t, s>0$. Letting $s \rightarrow 0^{+}$in (3.4) gives

$$
h\left(k x, k^{2} t\right)=k^{2} h(x, t)
$$

for all $x \in \mathbb{R}^{n}, t>0$. Replacing $s$ by $k^{2} s$ in (3.4) and then using (3.5), we obtain

$$
h(x, t+s)=h(x, t)
$$

for all $x \in \mathbb{R}^{n}, t, s>0$. This shows that $h(x, t)$ is independent with respect to the second variable. Thus, we see that $H(x):=h(x, t)$ satisfies (1.2). Using the induction argument on the dimension $n$, we verify that every continuous solution of (1.2) in $\mathbb{R}^{n}$ is a quadratic form

$$
H(x)=h(x, t)=\sum_{1 \leq i \leq j \leq n} a_{i j} x_{i} x_{j}
$$

where $a_{i j} \in \mathbb{C}$.

On the other hand, putting $x=y=0$ in (3.2) yields

$$
f\left(0, k^{2} t+s\right)=k^{2} f(0, t)+f(0, s)
$$

for all $t, s>0$. In view of (3.6), we verify that $\lim _{s \rightarrow 0^{+}} f(0, s)=0$ and

$$
f\left(0, k^{2} t\right)=k^{2} f(0, t)
$$

for all $t>0$. It follows from (3.6) and (3.7) that we see that $f(0, t)$ satisfies the Cauchy functional equation

$$
f(0, t+s)=f(0, t)+f(0, s)
$$

for all $t, s>0$. Given the continuity, we have

$$
f(0, t)=b t
$$

for some $b \in \mathbb{C}$. Therefore, we finally obtain

$$
f(x, t)=h(x, t)+f(0, t)=\sum_{1 \leq i \leq j \leq n} a_{i j} x_{i} x_{j}+b t
$$

for all $x \in \mathbb{R}^{n}, t>0$.

As a direct consequence of the above lemma, we present the general solution of the quadratic functional equation (1.3) in the spaces of generalized functions.

Theorem 3.3 Every solution u in $\mathcal{S}^{\prime}\left(\mathbb{R}^{n}\right)\left(\right.$ or $\mathcal{F}^{\prime}\left(\mathbb{R}^{n}\right)$, resp.) of Eq. (1.3) is the quadratic form

$$
u=\sum_{1 \leq i \leq j \leq n} a_{i j} x_{i} x_{j}
$$

for some $a_{i j} \in \mathbb{C}$. 
Proof Convolving the tensor product $E_{t}(\xi) E_{s}(\eta)$ of $n$-dimensional heat kernels in both sides of (1.3), we have

$$
\begin{aligned}
{\left[(u \circ A) *\left(E_{t}(\xi) E_{s}(\eta)\right)\right](x, y) } & =\left\langle u \circ A, E_{t}(x-\xi) E_{s}(y-\eta)\right\rangle \\
& =\left\langle u_{\xi}, k^{-n} \int E_{t}\left(x-\frac{\xi-\eta}{k}\right) E_{s}(y-\eta) d \eta\right\rangle \\
& =\left\langle u_{\xi}, k^{-n} \int E_{t}\left(\frac{k x+y-\xi-\eta}{k}\right) E_{s}(\eta) d \eta\right\rangle \\
& =\left\langle u_{\xi}, \int E_{k^{2} t}(k x+y-\xi-\eta) E_{s}(\eta) d \eta\right\rangle \\
& =\left\langle u_{\xi},\left(E_{k^{2} t} * E_{s}\right)(k x+y-\xi)\right\rangle \\
& =\left\langle u_{\xi}, E_{k^{2} t+s}(k x+y-\xi)\right\rangle \\
& =\tilde{u}\left(k x+y, k^{2} t+s\right)
\end{aligned}
$$

and similarly we get

$$
\begin{aligned}
& {\left[(u \circ B) *\left(E_{t}(\xi) E_{s}(\eta)\right)\right](x, y)=\tilde{u}\left(k x-y, k^{2} t+s\right),} \\
& {\left[(u \circ P) *\left(E_{t}(\xi) E_{s}(\eta)\right)\right](x, y)=\tilde{u}(x, t),} \\
& {\left[(u \circ Q) *\left(E_{t}(\xi) E_{s}(\eta)\right)\right](x, y)=\tilde{u}(y, s) .}
\end{aligned}
$$

Thus, (1.3) is converted into the classical functional equation

$$
\tilde{u}\left(k x+y, k^{2} t+s\right)+\tilde{u}\left(k x-y, k^{2} t+s\right)=2 k^{2} \tilde{u}(x, t)+2 \tilde{u}(y, s)
$$

for all $x, y \in \mathbb{R}^{n}, t, s>0$. We note that the Gauss transform $\tilde{u}$ is a $C^{\infty}$ function and so, by Lemma 3.2, the solution $\tilde{u}$ is of the form

$$
\tilde{u}(x, t)=\sum_{1 \leq i \leq j \leq n} a_{i j} x_{i} x_{j}+b t
$$

for some $a_{i j}, b \in \mathbb{C}$. By the heat kernel method, we obtain

$$
u=\sum_{1 \leq i \leq j \leq n} a_{i j} x_{i} x_{j}
$$

as $t \rightarrow 0^{+}$in (3.8).

\section{Stability in generalized functions}

In this section, we are going to solve the stability problem of (1.4). For the case of $k=1$ in (1.4), the result is known as follows.

Theorem 4.1 $([7,10])$ Suppose that u in $\mathcal{S}^{\prime}\left(\mathbb{R}^{n}\right)\left(\right.$ or $\mathcal{F}^{\prime}\left(\mathbb{R}^{n}\right)$, resp.) satisfies the inequality

$$
\|u \circ A+u \circ B-2 u \circ P-2 u \circ Q\| \leq \epsilon .
$$


Then there exists a unique quadratic form

$$
T(x)=\sum_{1 \leq i \leq j \leq n} a_{i j} x_{i} x_{j}
$$

such that

$$
\|u-T(x)\| \leq \frac{\epsilon}{2}
$$

We here need the following lemma to solve the stability problem of (1.4).

Lemma 4.2 Let $k$ be a fixed positive integer with $k \geq 2$. Suppose that $f: \mathbb{R}^{n} \times(0, \infty) \rightarrow \mathbb{C}$ is a continuous function satisfying the inequality

$$
\left\|f\left(k x+y, k^{2} t+s\right)+f\left(k x-y, k^{2} t+s\right)-2 k^{2} f(x, t)-2 f(y, s)\right\|_{L^{\infty}} \leq \epsilon .
$$

Then there exist a unique function $g(x, t)$ satisfying the quadratic-additive functional equation

$$
g\left(k x+y, k^{2} t+s\right)+g\left(k x-y, k^{2} t+s\right)=2 k^{2} g(x, t)+2 g(y, s)
$$

such that

$$
\|f(x, t)-g(x, t)\|_{L^{\infty}} \leq \frac{k^{2}+1}{2 k^{2}\left(k^{2}-1\right)} \epsilon .
$$

Proof Putting $x=y=0$ in (4.1) yields

$$
\left|f\left(0, k^{2} t+s\right)-k^{2} f(0, t)-f(0, s)\right| \leq \frac{\epsilon}{2}
$$

for all $t, s>0$. In view of (4.2), we see that

$$
c:=\limsup _{t \rightarrow 0^{+}} f(0, t)
$$

exists. Letting $t=t_{n} \rightarrow 0^{+}$so that $f\left(0, t_{n}\right) \rightarrow c$ in (4.2) gives

$$
|c| \leq \frac{\epsilon}{2 k^{2}}
$$

Putting $y=0$ and letting $s=s_{n} \rightarrow 0^{+}$so that $f\left(0, s_{n}\right) \rightarrow c$ in (4.1) we have

$$
\left|f\left(k x, k^{2} t\right)-k^{2} f(x, t)-c\right| \leq \frac{\epsilon}{2}
$$

for all $x \in \mathbb{R}^{n}, t>0$. Using (4.3), we can rewrite (4.4) as

$$
\left|\frac{f\left(k x, k^{2} t\right)}{k^{2}}-f(x, t)\right| \leq \frac{k^{2}+1}{2 k^{4}} \epsilon
$$


for all $x \in \mathbb{R}^{n}, t>0$. By the induction argument yields

$$
\left|\frac{f\left(k^{n} x, k^{2 n} t\right)}{k^{2 n}}-f(x, t)\right| \leq \frac{k^{2}+1}{2 k^{2}\left(k^{2}-1\right)} \epsilon
$$

for all $n \in \mathbb{N}, x \in \mathbb{R}^{n}, t>0$. We claim that the sequence $\left\{k^{-2 n} f\left(k^{n} x, k^{2 n} t\right)\right\}$ converges. Replacing $x$ by $k^{m} x$ and $t$ by $k^{2 m} t$ in (4.5), respectively, where $m \geq n$, we get

$$
\left|\frac{f\left(k^{m+n} x, k^{2(m+n)} t\right)}{k^{2(m+n)}}-\frac{f\left(k^{m} x, k^{2 m} t\right)}{k^{2 m}}\right| \leq \frac{k^{2}+1}{2 k^{2(m+1)}\left(k^{2}-1\right)} \epsilon
$$

Letting $n \rightarrow \infty$, by Cauchy convergence criterion, we see that the sequence $\left\{k^{-2 n} f\left(k^{n} x\right.\right.$, $\left.\left.k^{2 n} t\right)\right\}$ is a Cauchy sequence. We can now define a function $h: \mathbb{R}^{n} \times(0, \infty) \rightarrow \mathbb{C}$ by

$$
g(x, t):=\lim _{n \rightarrow \infty} \frac{f\left(k^{n} x, k^{2 n} t\right)}{k^{2 n}} .
$$

Letting $n \rightarrow \infty$ in (4.5) we obtain

$$
\|f(x, t)-g(x, t)\|_{L^{\infty}} \leq \frac{k^{2}+1}{2 k^{2}\left(k^{2}-1\right)} \epsilon .
$$

Replacing $x, y, t, s$ by $k^{n} x, k^{n} y, k^{2 n} t, k^{2 n} s$ in (4.1), dividing both sides by $k^{2 n}$ and letting $n \rightarrow \infty$ we have

$$
g\left(k x+y, k^{2} t+s\right)+g\left(k x-y, k^{2} t+s\right)=2 k^{2} g(x, t)+2 g(y, s)
$$

for all $x, y \in \mathbb{R}^{n}, t, s>0$. Next, we shall prove that $g$ is unique. Suppose that there exists another function $h: \mathbb{R}^{n} \times(0, \infty) \rightarrow \mathbb{C}$ such that $h$ satisfies (4.6) and (4.7). Since $g$ and $h$ satisfy (4.7), we see from Lemma 3.2 that

$$
g\left(k^{n} x, k^{2 n} t\right)=k^{2 n} g(x, t), \quad h\left(k^{n} x, k^{2 n} t\right)=k^{2 n} h(x, t)
$$

for all $n \in \mathbb{N}, x \in \mathbb{R}^{n}, t>0$. One gets from (4.6) that

$$
\begin{aligned}
& |g(x, t)-h(x, t)| \\
& \quad=k^{-2 n}\left|g\left(k^{n} x, k^{2 n} t\right)-h\left(k^{n} x, k^{2 n} t\right)\right| \\
& \quad \leq k^{-2 n}\left(\left|g\left(k^{n} x, k^{2 n} t\right)-f\left(k^{n} x, k^{2 n} t\right)\right|+\left|f\left(k^{n} x, k^{2 n} t\right)-h\left(k^{n} x, k^{2 n} t\right)\right|\right) \\
& \quad \leq \frac{k^{2}+1}{k^{2(n+1)}\left(k^{2}-1\right)} \epsilon
\end{aligned}
$$

for all $n \in \mathbb{N}, x \in \mathbb{R}^{n}, t>0$. Taking the limit as $n \rightarrow \infty$, we conclude that $g(x, t)=h(x, t)$ for all $x \in \mathbb{R}^{n}, t>0$.

We now state and prove the main theorem of this paper. 
Theorem 4.3 Suppose that u in $\mathcal{S}^{\prime}\left(\mathbb{R}^{n}\right)\left(\right.$ or $\mathcal{F}^{\prime}\left(\mathbb{R}^{n}\right)$, resp.) satisfies the inequality (1.4). Then there exists a unique quadratic form

$$
T(x)=\sum_{1 \leq i \leq j \leq n} a_{i j} x_{i} x_{j}
$$

such that

$$
\|u-T(x)\| \leq \begin{cases}\frac{\epsilon}{2}, & k=1, \\ \frac{\left(k^{2}+1\right) \epsilon}{2 k^{2}\left(k^{2}-1\right)}, & k \geq 2 .\end{cases}
$$

Proof As discussed above, it is done for the case of $k=1$. We assume that $k$ is a fixedpositive integer with $k \geq 2$. Convolving the tensor product $E_{t}(\xi) E_{s}(\eta)$ of $n$-dimensional heat kernels in both sides of (1.4), we have

$$
\left\|\tilde{u}\left(k x+y, k^{2} t+s\right)+\tilde{u}\left(k x-y, k^{2} t+s\right)-2 k^{2} \tilde{u}(x, t)-2 \tilde{u}(y, s)\right\|_{L^{\infty}} \leq \epsilon .
$$

By Lemma 4.2, there exists a unique function $g(x, t)$ satisfying the quadratic-additive functional equation

$$
g\left(k x+y, k^{2} t+s\right)+g\left(k x-y, k^{2} t+s\right)=2 k^{2} g(x, t)+2 g(y, s)
$$

such that

$$
\|\tilde{u}(x, t)-g(x, t)\|_{L^{\infty}} \leq \frac{k^{2}+1}{2 k^{2}\left(k^{2}-1\right)} \epsilon .
$$

It follows from Lemma 3.2 that $g(x, t)$ is of the form

$$
g(x, t)=\sum_{1 \leq i \leq j \leq n} a_{i j} x_{i} x_{j}+b t
$$

for some $a_{i j}, b \in \mathbb{C}$. Letting $t \rightarrow 0^{+}$in (4.8), we have

$$
\left\|u-\sum_{1 \leq i \leq j \leq n} a_{i j} x_{i} x_{j}\right\| \leq \frac{k^{2}+1}{2 k^{2}\left(k^{2}-1\right)} \epsilon .
$$

This completes the proof.

Remark 4.4 The resulting inequality in Theorem 4.3 implies that $u-T(x)$ is a measurable function. Thus, all of the solution $u$ in $\mathcal{S}^{\prime}\left(\mathbb{R}^{n}\right)$ (or $\mathcal{F}^{\prime}\left(\mathbb{R}^{n}\right)$, resp.) can be written uniquely in the form

$$
u=T(x)+\mu(x)
$$

where

$$
\|\mu\|_{L^{\infty}} \leq \begin{cases}\frac{\epsilon}{2}, & k=1, \\ \frac{\left(k^{2}+1\right) \epsilon}{2 k^{2}\left(k^{2}-1\right)}, & k \geq 2 .\end{cases}
$$




\section{Competing interests}

The author declares that they have no competing interests.

Received: 12 September 2011 Accepted: 6 August 2012 Published: 16 August 2012

\section{References}

1. Aczél, J, Dhombres, J: Functional Equations in Several Variables. Cambridge University Press, Cambridge (1989)

2. Baker, JA: Distributional methods for functional equations. Aequ. Math. 62, 136-142 (2001)

3. Borelli, C, Forti, GL: On a general Hyers-Ulam-stability result. Int. J. Math. Math. Sci. 18, 229-236 (1995)

4. Cholewa, PW: Remarks on the stability of functional equations. Aequ. Math. 27, 76-86 (1984)

5. Chung, J, Chung, S-Y, Kim, D: Une caractérisation de l'espace $\mathcal{S}$ de Schwartz. C. R. Math. Acad. Sci. Paris 316, 23-25 (1993)

6. Chung, J, Chung, S-Y, Kim, D: A characterization for Fourier hyperfunctions. Publ. Res. Inst. Math. Sci. 30, 203-208 (1994)

7. Chung, J: Stability of functional equations in the spaces of distributions and hyperfunctions. J. Math. Anal. Appl. 286, 177-186 (2003)

8. Chung, J, Lee, S: Some functional equations in the spaces of generalized functions. Aequ. Math. 65, 267-279 (2003)

9. Chung, J, Chung, S-Y, Kim, D: The stability of Cauchy equations in the space of Schwartz distributions. J. Math. Anal. Appl. 295, 107-114 (2004)

10. Chung, J: A distributional version of functional equations and their stabilities. Nonlinear Anal. 62, 1037-1051 (2005)

11. Chung, J: Stability of approximately quadratic Schwartz distributions. Nonlinear Anal. 67, 175-186 (2007)

12. Czerwik, S: On the stability of the quadratic mapping in normed spaces. Abh. Math. Semin. Univ. Hamb. 62, 59-64 (1992)

13. Czerwik, S: Functional Equations and Inequalities in Several Variables. World Scientific, River Edge (2002)

14. Găvruţa, P: A generalization of the Hyers-Ulam-Rassias stability of approximately additive mappings. J. Math. Anal. Appl. 184, 431-436 (1994)

15. Hörmander, L: The Analysis of Linear Partial Differential Operators I. Springer, Berlin (1983)

16. Hyers, DH: On the stability of the linear functional equation. Proc. Natl. Acad. Sci. USA 27, $222-224$ (1941)

17. Hyers, DH, Isac, G, Rassias, TM: Stability of Functional Equations in Several Variables. Birkhäuser, Boston (1998)

18. Jun, K-W, Lee, Y-H: On the Hyers-Ulam-Rassias stability of a pexiderized quadratic inequality. Math. Inequal. Appl. 4, 93-118 (2001)

19. Kannappan, P: Functional Equations and Inequalities with Applications. Springer, Berlin (2009)

20. Kim, KW, Chung, S-Y, Kim, D: Fourier hyperfunctions as the boundary values of smooth solutions of heat equations. Publ. Res. Inst. Math. Sci. 29, 289-300 (1993)

21. Lee, JR, An, JS, Park, C: On the stability of quadratic functional equations. Abstr. Appl. Anal. 2008, Art. ID 628178 (2008)

22. Lee, Y-S, Chung, S-Y: Stability of cubic functional equation in the spaces of generalized functions. J. Inequal. Appl. 2007, Art. ID 79893 (2007)

23. Matsuzawa, T: A calculus approach to hyperfunctions III. Nagoya Math. J. 118, 133-153 (1990)

24. Najati, A, Eskandani, GZ: A fixed point method to the generalized stability of a mixed additive and quadratic functional equation in Banach modules. J. Differ. Equ. Appl. 16, 773-788 (2010)

25. Rassias, TM: On the stability of the linear mapping in Banach spaces. Proc. Am. Math. Soc. 72, 297-300 (1978)

26. Saadati, R, Park, C: Non-Archimedian $\mathcal{L}$-fuzzy normed spaces and stability of functional equations. Comput. Math. Appl. 60, 2488-2496 (2010)

27. Sahoo, PK, Kannappan, P: Introduction to Functional Equations. CRC Press, Boca Raton (2011)

28. Schwartz, L: Théorie des distributions. Hermann, Paris (1966)

29. Skof, F: Local properties and approximation of operators. Rend. Semin. Mat. Fis. Milano 53, 113-129 (1983)

30. Trif, T: On the stability of a general gamma-type functional equation. Publ. Math. (Debr.) 60, 47-61 (2002)

31. Ulam, SM: Problems in Modern Mathematics. Wiley, New York (1964)

32. Wang, L: Intuitionistic fuzzy stability of a quadratic functional equation. Fixed Point Theory Appl. 2010, Art. ID 107182 (2010)

doi:10.1186/1029-242X-2012-177

Cite this article as: Lee: Stability of quadratic functional equations in tempered distributions. Journal of Inequalities and Applications 2012 2012:177.

\section{Submit your manuscript to a SpringerOpen ${ }^{\circ}$ journal and benefit from:}

- Convenient online submission

- Rigorous peer review

- Immediate publication on acceptance

Open access: articles freely available online

- High visibility within the field

- Retaining the copyright to your article 